\title{
New treatments and new choices for type 2 diabetes mellitus
}

\author{
D Bhatnagar \\ Consultant/Senior Lecturer in Diabetes \& Metabolism, Royal Oldham Hospital and University of Manchester Cardiovascular Research Group, \\ Oldham, UK
}

TITLE Liraglutide versus sitagliptin for patients with type 2 diabetes who did not have adequate glycaemic control with metformin: a 26-week, randomised, parallelgroup, open-label trial

AUTHORS Pratley RE, Nauck M, Bailey T et al, for the 1860-LIRA-DPP-4 Study Group.

JOURNAL Lancet 20I0; 375:|447-56. doi:I0.10I6/S0I40-6736(I0)60307-8

DECLARATION OF INTERESTS The author has received speaker fees and payment from manufacturers of insulin and other injectable and oral hypoglycaemic agents.
Correspondence to D Bhatnagar, Diabetes Centre, Royal Oldham Hospital, Rochdale Road, Oldham OLI 2JH, UK
tel. $+44(0) 1616278384$
e-mail
d.bhatnagar@manchester.ac.uk

\section{SUMMARY}

This randomised controlled trial funded by the makers of liraglutide explores whether injectable or oral agents acting on the incretin system should be used in patients with type 2 diabetes mellitus in subjects inadequately controlled on metformin alone and naïve to other hypoglycaemic agents. This was a multinational study carried out in a number of European countries, Canada and the USA. Subjects in all three groups (described below) were normotensive but obese with a body mass index of around $33 \mathrm{~kg} / \mathrm{m}^{2}$. They had moderate glycaemic control with mean glycated haemoglobin $\left(\mathrm{HbA}_{\mathrm{lc}}\right)$ levels of around $8.4 \%$. The mean duration of diabetes was less than 6.5 years.

Of 665 patients who entered the trial, 554 completed the 26-week study. There were three groups of subjects. One group received the dipeptidyl peptidase-4 (DPP4) inhibitor sitagliptin as a $100 \mathrm{mg}$ tablet daily and the other two groups received the injectable glucagon-like peptide-I (GLP-I) receptor agonist liraglutide (a GLP-I analogue). The latter two groups were made up of those who received $1.2 \mathrm{mg}$ and $1.8 \mathrm{mg}$ of liraglutide respectively.

After 26 weeks, mean decreases in $\mathrm{HbA}_{\mathrm{lc}}$ from baseline were $0.9 \%$ for the sitagliptin group, I. $2 \%$ for the liraglutide $1.2 \mathrm{mg}$ group and $1.5 \%$ for those on $1.8 \mathrm{mg}$ of liraglutide. About $28 \%$ of participants had a baseline $\mathrm{HbA}_{\mathrm{lc}}$ greater than $9.0 \%$ and the decreases in $\mathrm{HbA}_{\mathrm{lc}}$ were more pronounced in this cohort, with $1.2 \%$ for those on sitagliptin, $1.7 \%$ for those on $1.2 \mathrm{mg}$ liraglutide and $2.1 \%$ for those on $1.8 \mathrm{mg}$ liraglutide. Therefore, significantly more patients achieved the stated $\mathrm{HbA}_{\mathrm{lc}}$ targets of $<7.0 \%$ and $\leq 6.5 \%$ with liraglutide than with sitagliptin. At 26 weeks those on $1.8 \mathrm{mg}$ liraglutide lost most weight (-3.38 $\mathrm{kg})(95 \%$ confidence interval $[\mathrm{Cl}],-3.91$ to -2.84$)$ compared with $-2.86 \mathrm{~kg}(-3.39$ to -2.2$)$ for $1.2 \mathrm{mg}$ liraglutide and $-0.96 \mathrm{~kg}(-\mathrm{I} .50$ to -0.42$)$ for sitagliptin.
As judged by diabetes treatment satisfaction scores, no difference was perceived between the convenience of sitagliptin (oral) or liraglutide (injectable). There were more gastrointestinal side effects in those on liraglutide than on sitagliptin, with nausea being most marked in patients on liraglutide. There was no difference in hypoglycaemia reported in the three treatment groups.

\section{OPINION}

The discovery of incretin hormones came about following the observation that oral administration of glucose produced a more pronounced increase in insulin secretion than a similar intravenous glucose load.' This pointed to gut-related factors, and GLP-I and glucosedependent insulinotropic polypeptide appear to be the two key hormones. Glucagon-like peptide-I was developed as a pharmaceutical agent since it not only increased glucose-dependent insulin secretion but also suppressed glucagon secretion and decreased food intake. Two approaches were taken. One was to increase the naturally produced GLP-I. This was achieved by inhibiting the enzyme DPP-4 which rapidly inactivates GLP-I in the body. This class of drugs colloquially called 'gliptins' are administered orally and sitagliptin is an example. The other way to increase GLP-I is to produce GLP-I receptor agonists. These are injectable and liraglutide, a GLP-I analogue, is an example of this class of drugs.

Both drugs are relatively new so how does the physician choose which drug to use? This paper provides some of the answers, but not all. It is current practice to start all newly diagnosed patients with type 2 diabetes on metformin. The journey thereafter depends on a number of non-drug-related factors. Some patients will have been diagnosed early, whereas others will be discovered after a long period and both groups would have varying degrees of insulin resistance. Some would be more obese than others and the elderly would be more prone 
to hypoglycaemia. It is, therefore, prudent to individualise treatment rather than adding another oral drug or insulin when patients fail to achieve the recommended $\mathrm{HbA}_{\mathrm{lc}}$ targets of $7.5 \%$. This bland statement hides a number of hidden practical therapeutic issues. The currently used sulphonylurea and thiazolinedione drugs and insulin result in significant weight gain and produce more guilt feelings in patients who are generally already obese. Starting someone on insulin is perceived by both doctors and patients as a failure of treatment. Patients, of course, are reluctant to receive injections. The welcome introduction of a new class of drugs provides more choice for the specialist physician and general practitioner alike.

Both DPP-4 inhibitors and GLP-I receptor agonists increase insulin secretion in a glucose-dependent manner and are thought to improve beta cell function in animal models. ${ }^{2}$ It would be best to use drugs such as sitagliptin and liraglutide as early as possible or even as a combination with metformin at the time of diagnosis. What precludes such an approach is that the long-term side effects of these drugs are still unknown and they are expensive. The well-publicised debate and withdrawal of rosiglitazone would certainly advocate caution to such an approach. ${ }^{3}$ There are a number of dipeptidyl peptidase enzymes and the safety and efficacy of the gliptin drug depends on the selectivity of the compound for DPP-4 In animal studies the inhibition of dipeptidyl peptidases other than DPP-4 has produced skin lesions. A curious increase in upper respiratory tract infections has also been reported. With the GLP-I receptor agonists there have been reports of pancreatitis and a rise in serum calcitonin, although these findings are not borne out in collective registry data.

So how should the likes of sitagliptin and liraglutide be used? There are differences beyond the oral and injectable modes of administration. Liraglutide with its added benefit of producing weight loss is best used in subjects who are obese. Sitagliptin is perhaps best used in people who do not wish to inject. The gliptins are also useful in the elderly who are prone to hypoglycaemia, particularly with sulphonylurea drugs. Both drugs reduce $\mathrm{HbA}_{\mathrm{Ic}}$ by $\mathrm{I}-\mathrm{I} .5 \%$, a decrease not dissimilar to that seen with metformin. It is, therefore, asking too much of these agents to improve glycaemic control dramatically in those with poor glycaemic control.

There are marginal benefits in using these drugs in those already on two or three oral agents as glycaemic control is unlikely to improve substantially. Such patients are best treated with intensive lifestyle advice and insulin. The expected benefits of improved glycaemic control following weight loss induced by GLP-I receptor agonists are not evident in all patients. It may well be that these are patients who have little beta cell reserve left. In this context the paper under review no doubt confirms the benefits of liraglutide over sitagliptin, but given the numbers in the study it does not provide firm answers on whether there was a difference in effect between the two agents in relation to the duration of diabetes.

More potent DPP-4 inhibitors that also produce weight loss are in the pipeline and once-weekly injections of GLP-I receptor agonists are currently being developed. Combinations of DPP-4 inhibitors and metformin are already available and combinations of long-acting insulin and GLP-I receptor agonists are being considered. It would be interesting to know how these agents would be used clinically, particularly the once-a-week injectables. Does the patient who has already had his weekly GLP-I injection refuse an invitation to a meal out or a birthday party that week? After a long time there are exciting times ahead for people with diabetes, with new classes of drugs and new ways and combinations of drugs to use. These developments will help the physician to individualise treatment and bring some hope and choice to patients who struggle with their lifestyle everyday.

\section{REFERENCES}

I Drucker DJ, Nauck MA. The incretin system: glucagon-like peptide-I receptor agonists and dipeptidyl peptidase-4 inhibitors in type 2 diabetes. Lancet 2006; 368:1696-705. doi: 10.1016/S0I406736(06)69705-5
2 Neumiller JJ, Wood L, Campbell RK. Dipeptidyl peptidase-4 inhibitors for the treatment of type 2 diabetes mellitus. Pharmacotherapy 2010; 30:463-84. doi: 10.1592/phco.30.5.463

3 Nissen SE. The rise and fall of rosiglitazone. Eur Heart J 2010; 3I:773-6. doi:I0.1093/eurheartj/ehq0I6 